\begin{tabular}{cc|c}
\hline Tar. Bil. Der. & Tarm Bilimleri Dergisi & Journal of Agricultural Sciences \\
& $\begin{array}{c}\text { Dergi web sayfası: } \\
\text { www.agri.ankara.edu.tr/dergi }\end{array}$ & Journal homepage: \\
& www.agri.ankara.edu.tr/journal
\end{tabular}

\title{
Identification of Hordeum spontaneum Genotypes Resistant to Net Blotch Disease
}

\author{
Arzu ÇELİK OĞUZ ${ }^{a}$, Aziz KARAKAYA ${ }^{a}$, Rukiye MURAT DURAN ${ }^{b}$, Kürşad ÖZBEK ${ }^{b}$ \\ a Ankara University, Faculty of Agriculture, Department of Plant Protection, Dışkapı, Ankara, TURKEY \\ ${ }^{\boldsymbol{b}}$ Field Crops Central Research Institute, Şehit Cem Ersever Caddesi, No: 9-11, Yenimahalle, Ankara, TURKEY
}

\section{ARTICLE INFO}

Research Article DOI: 10.15832/ankutbd.539014

Corresponding Author: Aziz KARAKAYA, E-mail: karakaya@agri.ankara.edu.tr, Tel: +90 (312) 5961258

Received: 13 December 2017, Received in Revised Form: 17 March 2018, Accepted: 17 April 2018

\begin{abstract}
Wild barley (Hordeum spontaneum) is a progenitor of cultivated barley and naturally grows in Turkey. H. spontaneum genotypes possess superior characteristics for biotic and abiotic stress tolerance factors. In this study, 3 virulent Pyrenophora teres f. maculata and 3 virulent $P$. teres f. teres isolates were tested under greenhouse conditions in order to find net blotch resistant $H$. spontaneum genotypes. A total of $104 H$. spontaneum genotypes were used. Twenty-six H. spontaneum genotypes which corresponded to $25 \%$ of the genotypes (genotypes numbered $8,13,14,16,22,24$, $27,31,37,44,47,54,58,62,65,66,69,74,78,81,89,94,99,102,104$ and 107) exhibited reactions classified in the resistant group to 3 virulent $P$. teres f. maculata isolates. Eight $H$. spontaneum genotypes which corresponded to $7.6 \%$ of the genotypes (genotypes numbered 24, 27, 29, 33, 44, 54, 89 and 94) exhibited reactions classified in the resistant group to 3 virulent $P$. teres f. teres isolates. Six $H$. spontaneum genotypes which corresponded to $5.7 \%$ of the genotypes (genotypes numbered 24, 27, 44, 54, 89 and 94) exhibited reactions in the resistant group to both 6 virulent $P$. teres f. teres and $P$. teres f. maculata isolates. In addition, a considerable number of genotypes exhibited resistant group reactions to one or two isolates of both forms of the pathogen. These genotypes could be used for developing net blotch resistant barley cultivars.
\end{abstract}

Keywords: Barley; Disease resistance; Hordeum spontaneum; Net blotch; Pyrenophora teres

(C) Ankara Üniversitesi Ziraat Fakültesi

\section{Introduction}

Wild barley (Hordeum spontaneum, syn: Hordeum vulgare subsp. spontaneum) is a progenitor of cultivated barley and naturally grows in Turkey (Kün 1996; Karakaya et al 2016). Hordeum spontaneum could hybridize with cultivated barley (Hordeum vulgare) and is an important plant for developing disease resistant barley cultivars. Wild barleys and barley landraces possess superior characteristics for abiotic and biotic stress tolerance factors (Ceccarelli \& Grando 2000; Karakaya et al 2016; Çelik \& Karakaya 2017). Because of these characteristics, it is advised to preserve $H$. spontaneum genotypes in situ and ex situ conditions for future research (Nevo 2012).

China, India, Central Asia, Near East, Mediterranean region, Ethiopia, Southern Mexico and Central and South America are eight main 
regions in the world considered as plant gene centers. Turkey has a rich genetical diversity due to its location. Turkey is located at the intersection of the Mediterranean and the Near East gene centers, and it is on the historical migration and transportation routes of China, India, Central Asia and Ethiopia gene centers. In addition, Fertile Crescent region which includes Turkey's Southeastern Anatolia region is known as the region where barley, wheat, lentil, hard-seeded fruit and olive are cultivated for the first time (Vavilov 1951). In addition, Jakob et al (2014) reported that Levant, Turkey and east of Turkey are three main regions of wild barley $(H$. spontaneum) populations. Wild barleys including $H$. spontaneum are commonly grown under natural conditions in Turkey (Karakaya et al 2016).

Net blotch is caused by the fungus Pyrenophora teres that belongs to ascomycota. Anamorphic stage of the fungus is named as Drechslera teres. Two biotypes of the fungus are recognized. Pyrenophora teres f. maculata incites the spot form and Pyrenophora teres f. teres incites the net form of the disease (Karakaya \& Akyol 2006; Liu et al 2011). The disease is commonly reported from different parts of the world and reduces the yield and quality of barley considerably (Mathre 1982; Liu et al 2011; Karakaya et al 2014).

In this study, 3 virulent $P$. teres $\mathrm{f}$. maculata $(P t m)$ and 3 virulent $P$. teres f. teres $(P t t)$ isolates were tested under greenhouse conditions in order to find net blotch resistant $H$. spontaneum genotypes. A total of $104 \mathrm{H}$. spontaneum genotypes were used. An abstract of this study has been published previously (Çelik Oğuz et al 2017).

\section{Materials and Methods}

\subsection{Experimental materials}

In this study, 107 wild barley (H. spontaneum) genotypes that collected from various parts of Turkey and conserved by Field Crops Central Research Institute (Ankara, Turkey) were used. The seeds of these $H$. spontaneum genotypes were multiplied from single heads. Out of 107 genotypes,
104 provided the sufficient amount of seeds and were included in this study. No sufficient seeds were obtained from genotypes No: 4, No: 15 and No: 41. Three Ptm isolates and $3 \mathrm{Ptt}$ isolates that were found to be the most virulent ones in the study by Çelik Oğuz (2015) were used in determination of seedling stage resistance of $104 \mathrm{H}$. spontaneum genotypes under greenhouse conditions.

\subsection{Treatments}

Sterile mixtures of soil, sand and organic substances $(60,20,20 ; \mathrm{v} / \mathrm{v} / \mathrm{v}$, respectively) were placed in plastic pots with diameters of 7 centimeters and depending on the quantity of seeds of genotypes, 5-10 seeds were placed to the pots. The pots were maintained under greenhouse conditions. Inoculation was performed at growth stages 12-13 (Zadoks et al 1974). The inoculum was prepared from cultures grown on Potato Dextrose Agar maintained at $16-23 \pm 2{ }^{\circ} \mathrm{C}$ night/day with a $10 \mathrm{~h} / 14 \mathrm{~h}$ dark/light period. In order to prepare inoculum, mycelia were harvested from Petri dishes using a no. 12 brush and concentration of inoculum was adjusted to $15-20 \times 10^{4}$ mycelial parts/ $\mathrm{ml}$ (Douiyssi et al 1998; Taşkoparan \& Karakaya 2009; Usta et al 2014; Yazic1 et al 2015). One drop of Tween 20 was added to each $100 \mathrm{~mL}$ of inoculum (Aktaş 1995). Inoculum was sprayed onto barley seedlings using a hand sprayer and all leaves were covered with inoculum. The greenhouse temperature ranged between $18-23 \pm 1{ }^{\circ} \mathrm{C}$ night/day with a $10 \mathrm{~h} / 14$ $\mathrm{h}$ dark/light period. Plants were kept covered with nylon in transparent boxes with moist lids for $76 \mathrm{~h}$ following inoculation. Then, plants were maintained for another $48 \mathrm{~h}$ with the nylon uncovered and the ventilation of the boxes activated. There were three replications.

\subsection{Evaluation of the disease}

Plant evaluations were carried out seven days later following inoculation. For evaluation, scales developed for both forms of net blotch by Tekauz (1985) were used. Plant evaluations were based on lesion size, morphology, necrosis and chlorosis. Scale values of 1,2 and 3 were considered as resistant group in this study. In the scale for the spot 
form of net blotch, seven numerical classes were defined $(1=\mathrm{R}$ : resistant, $2=\mathrm{R}$ : resistant to MR: moderately resistant, $3=\mathrm{MR}$ : moderately resistant, 5= MR: moderately resistant to MS: moderately susceptible, $7=$ MS: moderately susceptible, $8=$ MS: moderately susceptible to S: susceptible, and $9=\mathrm{S}$ : susceptible). In net form of net blotch scale ten numerical classes were defined $(1=\mathrm{R}$ : resistant, $2=\mathrm{R}$ : resistant to MR: moderately resistant, $3=\mathrm{MR}$ : moderately resistant, $4=\underline{\text { MR: moderately resistant }}$ to MS: moderately susceptible, $5=$ MR: moderately resistant to MS: moderately susceptible, $6=\mathrm{MR}$ : moderately resistant to $\underline{\mathrm{MS}}$ : moderately susceptible, $7=$ MS: moderately susceptible, $8=$ MS: moderately susceptible to S: susceptible, $9=\mathrm{S}$ : susceptible, and $10=$ VS: very susceptible). Resistant or moderately resistant genotypes have small net blotch lesions. Moderately susceptible or susceptible genotypes have chlorotic zones surrounding the necrotic areas and coalescence of these areas and death of leaves can occur.

\subsection{Data analysis}

Experiment was carried out using randomized block design with three replications. Data were square root transformed before statistical analysis. Separate two way analysis of variance was performed for each isolate and means of responses of $H$. spontaneum genotypes were separated by Least Significant Difference (LSD) test. Statistical tests were accomplished using JMP software (version 11; SAS Institute).

\section{Results and Discussion}

Seedling resistance reactions of 104 wild barley genotypes to 3 virulent Ptm isolates and 3 virulent $P t t$ isolates were determined. Analysis of variance revealed significant differences among the $H$. spontaneum genotypes $(\mathrm{P}<0.01)$. Response of the genotypes ranged between resistant (scale value 1) and susceptible (scale value 9) (Table 1). Thirty-nine, 9 and 2 genotypes exhibited moderately resistant, resistant-moderately resistant and resistant reactions to Ptm isolate GPS 263 PTM, respectively. Thirtysix and 3 genotypes showed moderately resistant and resistant-moderately resistant reactions to $\mathrm{Ptm}$ isolate 13-179 PTM, respectively. Fifty-three, 9 and 3 genotypes exhibited moderately resistant, resistant-moderately resistant and resistant reactions to Ptm isolate 13-167 PTM, respectively.

Twenty-six $H$. spontaneum genotypes which corresponded to $25 \%$ of the genotypes (genotypes numbered $8,13,14,16,22,24,27,31,37,44,47$, $54,58,62,65,66,69,74,78,81,89,94,99,102$, 104 and 107) exhibited reactions classified in the resistant group to 3 virulent $P$ tm isolates.

Nine and 1 genotypes showed moderately resistant and resistant reactions to Ptt isolate GPS 18 PTT, respectively. Thirteen, 6 and 2 genotypes exhibited moderately resistant, resistant-moderately resistant and resistant reactions to Ptt isolate UHK 77 PTT, respectively. Twenty-one and 8 genotypes exhibited moderately resistant and resistantmoderately resistant reactions to $P t t$ isolate $13-130$ PTT, respectively.

Eight $H$. spontaneum genotypes which corresponded to $7.6 \%$ of the genotypes (genotypes numbered 24, 27, 29, 33, 44, 54, 89 and 94) exhibited reactions classified in the resistant group to 3 virulent $P t t$ isolates.

Six H. spontaneum genotypes which corresponded to $5.7 \%$ of the genotypes (genotypes numbered 24, 27, 44, 54, 89 and 94) exhibited reactions in the resistant to moderately resistant group range (Tekauz (1985) scale 1 to 3 ) to both 6 virulent Ptt and Ptm isolates. In addition, a considerable number of genotypes exhibited resistant to moderately resistant reactions to one or two isolates of both forms of the pathogen (Table 1).

Wild barleys are important resistance sources for controlling biotic and abiotic stress factors. Finding disease resistant wild barley genotypes facilitate disease resistance studies. In this current study, we determined $H$. spontaneum genotypes resistant to both forms of $P$. teres.

There are limited studies related to reactions of $H$. spontaneum genotypes to net blotch disease. In a study conducted by Kopahnke (1998), 770 
Table 1- Seedling reactions of 104 Hordeum spontaneum genotypes to 3 virulent Pyrenophora teres f. maculata isolates and 3 virulent Pyrenophora teres f. teres isolates. Means not connected by same letter are significantly different $(\mathrm{P}<0.01)$

\begin{tabular}{|c|c|c|c|c|c|c|c|c|c|c|c|c|}
\hline \multirow{2}{*}{$\begin{array}{l}\text { Isolate } \\
\text { Genotype }\end{array}$} & \multicolumn{2}{|c|}{ GPS 263} & \multicolumn{2}{|c|}{$13-179$} & \multicolumn{2}{|c|}{$13-167$} & \multicolumn{2}{|c|}{ GPS 18} & \multicolumn{2}{|c|}{$U H K ~ 77$} & \multicolumn{2}{|c|}{$13-130$} \\
\hline & \multicolumn{2}{|c|}{$P T M^{*}$} & \multicolumn{2}{|c|}{ PTM* } & \multicolumn{2}{|c|}{$P T M^{*}$} & & $T T^{* *}$ & & $T T^{* *}$ & & $T T^{* *}$ \\
\hline 1 & 5 cde & MR-MS & 5 bcd & MR-MS & 3 cde & MR & 4 fghı & MR-MS & $4 \mathrm{jjk}$ & MR-MS & 6 bcde & MR-MS \\
\hline 2 & 3 efg & MR & $5 \mathrm{bcd}$ & MR-MS & $5 a b c$ & MR-MS & 5 efgh & $\overline{\mathrm{MR}}-\mathrm{MS}$ & 5 ghı & $\overline{\mathrm{MR}}-\mathrm{MS}$ & 6 cdef & MR- $\overline{\mathrm{MS}}$ \\
\hline 3 & $3 \mathrm{def}$ & MR & 5 bcd & MR-MS & 5 bcd & MR-MS & 6 abcd & MR-MS & $5 \mathrm{fgh}$ & MR-MS & 6 abcd & MR-MS \\
\hline 4 & Not en & igh seeds & & & & & & & & & & \\
\hline 5 & $7 \mathrm{abc}$ & $\mathrm{MS}$ & $5 \mathrm{bc}$ & MR-MS & $5 \mathrm{abc}$ & MR-MS & 4 ghij & MR-MS & $4 \mathrm{ljk}$ & MR-MS & 5 efgh & MR-MS \\
\hline 6 & 3 def & MR & $5 \mathrm{bcd}$ & MR-MS & 3 cde & MR & 4 hijk & $\overline{\mathrm{MR}}-\mathrm{MS}$ & $4 \mathrm{jjk}$ & $\overline{\mathrm{MR}}-\mathrm{MS}$ & 4 hijk & MR-MS \\
\hline 7 & $5 \mathrm{bcd}$ & MR-MS & 5 bcd & MR-MS & 3 cde & MR & 6 bcde & MR-MS & $7 \mathrm{bc}$ & $\overline{\mathrm{MS}}$ & 6 bcde & MR-MS \\
\hline 8 & 3 efg & MR & 3 de & MR & $2 \mathrm{gh}$ & R-MR & 4 ghij & MR-MS & $6 \mathrm{efg}$ & MR-MS & 5 defg & MR-MS \\
\hline 9 & $5 \mathrm{cde}$ & MR-MS & $5 \mathrm{bcd}$ & MR-MS & $3 \mathrm{efg}$ & MR & 6 bcde & $\overline{\mathrm{MR}}-\mathrm{MS}$ & $8 \mathrm{ab}$ & MS- $\overline{\mathrm{S}}$ & 5 defg & MR-MS \\
\hline 10 & $7 \mathrm{abc}$ & MS & 3 cde & MR & $5 \mathrm{bcd}$ & MR-MS & 4 ghıj & MR-MS & $4 \mathrm{ijk}$ & MR-MS & $4 \mathrm{jjk}$ & MR-MS \\
\hline 11 & 5 cde & MR-MS & 3 cde & MR & 3 def & MR & $6 \mathrm{abc}$ & MR-MS & $7 \mathrm{bc}$ & $\mathrm{MS}$ & $8 \mathrm{a}$ & MS-S \\
\hline 12 & 3 efg & MR & $3 \mathrm{de}$ & MR & $5 \mathrm{bcd}$ & MR-MS & 6 bcde & MR-MS & $4 \mathrm{jjk}$ & MR-MS & 5 efgh & MR-MS \\
\hline 13 & 3 efg & MR & $3 \mathrm{de}$ & MR & 3 def & MR & 3 defg & $\underline{\mathrm{MR}}$ & $4 \mathrm{jkl}$ & MR-MS & $3 \mathrm{lmn}$ & $\underline{\mathrm{MR}}$ \\
\hline 14 & $2 \mathrm{fgh}$ & R-MR & $3 \mathrm{de}$ & MR & 3 def & MR & 4 fghı & MR-MS & $3 \mathrm{lmn}$ & $\mathrm{MR}$ & 4 ghij & MR-MS \\
\hline 15 & Not er & igh seeds & & & & & & & & & & \\
\hline 16 & 3 efg & MR & $3 \mathrm{de}$ & MR & $2 \mathrm{fgh}$ & R-MR & 6 bcde & MR-MS & 6 defg & MR-MS & 4 ghij & MR-MS \\
\hline 17 & $7 \mathrm{abc}$ & MS & $7 \mathrm{ab}$ & MS & $5 \mathrm{bcd}$ & MR-MS & 5 defg & MR-MS & $6 \mathrm{cdef}$ & MR-MS & 5 fghı & MR-MS \\
\hline 18 & $7 \mathrm{abc}$ & MS & $5 \mathrm{abc}$ & MR-MS & 3 cde & MR & 4 fghı & MR-MS & 5 efg & MR-MS & 5 fghı & MR-MS \\
\hline 19 & $5 \mathrm{abc}$ & MR-MS & $5 \mathrm{bc}$ & MR-MS & $5 \mathrm{abc}$ & MR-MS & 5 cdef & MR-MS & $7 \mathrm{~cd}$ & MS & $7 \mathrm{ab}$ & MS \\
\hline 20 & 5 cde & MR-MS & $5 \mathrm{bcd}$ & MR-MS & 3 def & MR & $6 \mathrm{abc}$ & MR-MS & 5 efg & MR-MS & 4 ghij & MR-MS \\
\hline 21 & $7 \mathrm{abc}$ & MS & 3 cde & MR & 3 def & MR & 6 bcde & MR- $\overline{\mathrm{MS}}$ & $5 \mathrm{fgh}$ & MR-MS & $4 \mathrm{jjk}$ & $\overline{\mathrm{MR}}-\mathrm{MS}$ \\
\hline 22 & $3 \mathrm{fgh}$ & MR & $3 \mathrm{de}$ & MR & 3 def & MR & 5 cdef & MR-MS & 6 cdef & MR-MS & 5 defg & MR-MS \\
\hline 23 & 5 cde & MR-MS & 3 cde & MR & $2 \mathrm{gh}$ & R-MR & 5 defg & MR-MS & 2 no & R-MR & 6 abcd & MR-MS \\
\hline 24 & 3 def & MR & $2 \mathrm{e}$ & R-MR & 2 efgh & R-MR & $2 \mathrm{~m}$ & R-MR & 20 & R-MR & $3 \mathrm{klm}$ & MR \\
\hline 25 & 5 bcd & MR-MS & 5 bcd & MR-MS & 5 bcd & MR-MS & 5 efgh & MR-MS & 6 defg & MR-MS & $5 \mathrm{efgh}$ & MR-MS \\
\hline 26 & $7 \mathrm{abc}$ & MS & $5 \mathrm{bc}$ & MR-MS & 3 cde & MR & 4 fghı & MR-MS & $4 \mathrm{rjk}$ & MR- $\overline{\mathrm{MS}}$ & $3 \mathrm{klm}$ & MR \\
\hline 27 & 3 efg & MR & $3 \mathrm{de}$ & MR & 2 efgh & R-MR & $31^{\circ}$ & $\overline{\mathrm{MR}}$ & $3 \mathrm{lmn}$ & $\overline{\mathrm{MR}}$ & $3 \mathrm{jkl}$ & MR \\
\hline 28 & $5 \mathrm{bcd}$ & MR-MS & $5 \mathrm{bcd}$ & MR-MS & 3 cde & $\mathrm{MR}$ & 5 defg & MR-MS & $5 \mathrm{fgh}$ & MR-MS & $7 \mathrm{abc}$ & MS \\
\hline 29 & 3 efg & MR & $3 \mathrm{de}$ & MR & 5 bcd & MR-MS & 31 & MR & $1 \mathrm{p}$ & $\mathrm{R}$ & $3 \mathrm{klm}$ & MR \\
\hline 30 & $7 \mathrm{abc}$ & MS & $5 \mathrm{abc}$ & MR-MS & 3 efg & MR & 4 ghijk & MR-MS & 6 defg & MR-MS & 5 efgh & MR-MS \\
\hline 31 & 3 def & MR & $3 \mathrm{de}$ & $\mathrm{MR}$ & $3 \mathrm{efg}$ & MR & 6 bcde & MR-MS & 6 defg & MR-MS & 6 bcde & MR-MS \\
\hline 32 & 3 efg & MR & $5 \mathrm{bcd}$ & MR-MS & $3 \mathrm{cde}$ & MR & 5 efgh & MR-MS & $6 \mathrm{cde}$ & MR-MS & $7 \mathrm{abc}$ & MS \\
\hline 33 & $2 \mathrm{fgh}$ & R-MR & $3 \mathrm{de}$ & MR & $5 \mathrm{bcd}$ & MR-MS & 3 1jkl & MR & $3 \mathrm{klm}$ & MR & $3 \mathrm{lmn}$ & MR \\
\hline 34 & 5 cde & MR-MS & $5 \mathrm{bcd}$ & MR-MS & $5 \mathrm{ab}$ & MR-MS & 6 abcd & MR-MS & $4 \mathrm{jjk}$ & MR-MS & 4 ghij & MR-MS \\
\hline 35 & $7 \mathrm{abc}$ & MS & $5 \mathrm{abc}$ & MR-MS & $5 \mathrm{abc}$ & MR-MS & $7 \mathrm{ab}$ & MS & $5 \mathrm{gh} 1$ & MR-MS & 6 bcde & MR-MS \\
\hline 36 & $5 \mathrm{bcd}$ & MR-MS & $5 \mathrm{bc}$ & MR-MS & $5 \mathrm{abc}$ & MR-MS & 6 bcde & MR-MS & 6 cdef & MR-MS & $6 \mathrm{cdef}$ & MR-MS \\
\hline 37 & 3 efg & MR & $3 \mathrm{de}$ & MR & 3 def & MR & 4 hijk & MR-MS & $3 \mathrm{mn}$ & MR & $3 \mathrm{lmn}$ & MR \\
\hline 38 & $5 \mathrm{cde}$ & MR-MS & 3 cde & MR & 3 cde & MR & 6 abcd & MR-MS & 6 cdef & MR-MS & 6 abcd & MR-MS \\
\hline 39 & $5 \mathrm{bcd}$ & MR-MS & $5 \mathrm{bcd}$ & MR-MS & 3 def & MR & 6 bcde & MR-MS & $6 \mathrm{cdef}$ & MR-MS & 5 efgh & MR-MS \\
\hline 40 & $7 \mathrm{abc}$ & MS & $5 \mathrm{abc}$ & MR-MS & $5 a b c$ & MR-MS & 6 bcde & MR-MS & $3 \mathrm{mn}$ & MR & 5 fghi & MR-MS \\
\hline 41 & Not en & igh seeds & & & & & & & & & & \\
\hline 42 & 3 efg & MR & 5 bcd & MR-MS & 3 def & MR & 6 bcde & MR-MS & $3 \mathrm{klm}$ & MR & $3 \mathrm{lmn}$ & MR \\
\hline 43 & $7 \mathrm{abc}$ & MS & $5 \mathrm{bc}$ & MR-MS & 3 def & MR & 6 bcde & MR-MS & $3 \mathrm{lmn}$ & MR & 2 no & R-MR \\
\hline 44 & 3 efg & MR & $3 \mathrm{de}$ & MR & 3 def & MR & 3 ijkl & MR & 2 no & R-MR & $3 \mathrm{lmn}$ & MR \\
\hline 45 & 5 cde & MR-MS & 5 bcd & MR-MS & 3 def & MR & 4 fghı & MR-MS & 6 defg & MR-MS & $3 \mathrm{lmn}$ & MR \\
\hline 46 & $5 \mathrm{abc}$ & MR-MS & $5 \mathrm{bc}$ & MR-MS & $5 \mathrm{bcd}$ & MR-MS & 5 defg & MR-MS & $7 \mathrm{~cd}$ & MR-MS & $3 \mathrm{lmn}$ & MR \\
\hline 47 & 3 efg & MR & $3 \mathrm{de}$ & MR & 3 efg & MR & 6 bcde & MR-MS & 2 no & R-MR & $2 \mathrm{mno}$ & R-MR \\
\hline 48 & $7 \mathrm{abc}$ & MS & $5 \mathrm{abc}$ & MR-MS & $5 \mathrm{bcd}$ & MR-MS & 6 bcde & MR-MS & $4 \mathrm{jjk}$ & MR-MS & $3 \mathrm{lmn}$ & MR \\
\hline 49 & $7 \mathrm{abc}$ & MS & $5 \mathrm{bc}$ & MR-MS & 3 def & $\mathrm{MR}$ & 4 hijk & MR-MS & $5 \mathrm{fgh}$ & MR-MS & $4 \mathrm{ijk}$ & MR-MS \\
\hline 50 & $7 \mathrm{abc}$ & MS & $5 \mathrm{bc}$ & MR-MS & 3 def & MR & 4 hijkl & MR-MS & $5 \mathrm{gh} 1$ & MR-MS & $4 \mathrm{jjk}$ & MR-MS \\
\hline 51 & 5 cde & MR-MS & 5 bcd & MR-MS & $5 \mathrm{bcd}$ & MR-MS & $6 \mathrm{abcd}$ & MR-MS & $6 \mathrm{cdef}$ & MR-MS & 6 cdef & MR-MS \\
\hline 52 & 3 efg & $\mathrm{MR}$ & 5 bcd & MR-MS & 5 bcd & MR-MS & 6 bcde & MR-MS & 6 cde & MR-MS & 6 bcde & MR-MS \\
\hline 53 & 3 efg & MR & 5 bcd & MR-MS & 5 bcd & MR-MS & 6 bcde & MR-MS & $5 \mathrm{fgh}$ & MR-MS & $4 \mathrm{ijk}$ & MR-MS \\
\hline 54 & $2 \mathrm{fgh}$ & R-MR & $3 \mathrm{de}$ & MR & $1 \mathrm{~h}$ & $\mathrm{R}$ & $3 \mathrm{kl}$ & MR & 2 no & R-MR & $2 \mathrm{mno}$ & $\overline{\mathrm{R}-\mathrm{M}}$ \\
\hline 55 & $3 \mathrm{efg}$ & MR & $5 \mathrm{bcd}$ & MR-MS & $5 \mathrm{bcd}$ & MR-MS & 6 bcde & MR-MS & $5 \mathrm{efg}$ & MR-MS & $3 \mathrm{lmn}$ & MR \\
\hline 56 & 5 cde & MR-MS & $3 \mathrm{de}$ & MR & 3 def & MR & 6 bcde & MR-MS & $4 \mathrm{ijk}$ & MR-MS & $3 \mathrm{jkl}$ & MR \\
\hline 57 & 3 def & MR & $5 \mathrm{abc}$ & MR-MS & $5 \mathrm{ab}$ & MR-MS & 6 bcde & MR-MS & $4 \mathrm{jkl}$ & MR-MS & 6 bcde & MR-MS \\
\hline 58 & 3 efg & MR & $3 \mathrm{de}$ & MR & 3 def & MR & 4 fghı & MR-MS & 6 cde & MR-MS & $4 \mathrm{rjk}$ & MR-MS \\
\hline 59 & $2 \mathrm{fgh}$ & R-MR & 5 bcd & MR-MS & 3 def & MR & 4 hijk & MR-MS & 6 cdef & MR-MS & $2 \mathrm{mno}$ & R-MR \\
\hline 60 & $5 \mathrm{bcd}$ & MR-MS & $5 \mathrm{bcd}$ & MR-MS & $3 \mathrm{def}$ & MR & $6 \mathrm{abcd}$ & $\overline{\mathrm{MR}}-\mathrm{MS}$ & $9 a$ & $\mathrm{~S}$ & 6 abcd & MR-MS \\
\hline 61 & $2 \mathrm{fgh}$ & R-MR & $5 \mathrm{bcd}$ & MR-MS & 3 cde & MR & 5 defg & MR-MS & $5 \mathrm{fgh}$ & MR-MS & 6 cdef & MR-MS \\
\hline 62 & $1 \mathrm{~h}$ & $\mathrm{R}$ & $3 \mathrm{de}$ & MR & $2 \mathrm{fgh}$ & R-MR & 6 bcde & MR-MS & $7 \mathrm{Cd}$ & MS & 5 efgh & MR-MS \\
\hline 63 & 3 def & MR & $5 \mathrm{bcd}$ & MR-MS & 3 cde & MR & 6 bcde & MR-MS & $4 \mathrm{ijk}$ & MR-MS & 4 hijk & MR-MS \\
\hline 64 & 3 efg & MR & $5 \mathrm{bcd}$ & MR-MS & 5 bcd & MR-MS & 4 fghı & MR-MS & $4 \mathrm{jkl}$ & MR-MS & $3 \mathrm{klm}$ & $\mathrm{MR}$ \\
\hline
\end{tabular}


Table 1- (continued)- Seedling reactions of 104 Hordeum spontaneum genotypes to 3 virulent Pyrenophora teres f. maculata isolates and 3 virulent Pyrenophora teres f. teres isolates. Means not connected by same letter are significantly different $(\mathrm{P}<\mathbf{0 . 0 1})$

\begin{tabular}{|c|c|c|c|c|c|c|c|c|c|c|c|c|}
\hline \multirow{2}{*}{$\begin{array}{l}\text { Isolate } \\
\text { Genotype } \\
65\end{array}$} & \multicolumn{2}{|c|}{$\begin{array}{c}G P S 263 \\
P T M^{*}\end{array}$} & \multicolumn{2}{|c|}{$\begin{array}{l}13-179 \\
P T M^{*}\end{array}$} & \multicolumn{2}{|c|}{$\begin{array}{l}\text { 13-167 } \\
P T M^{*}\end{array}$} & \multicolumn{2}{|c|}{$\begin{array}{l}\text { GPS 18 } \\
P T T^{* *}\end{array}$} & \multicolumn{2}{|c|}{$\begin{array}{l}U H K \text { 77 } \\
P T T^{* *}\end{array}$} & \multicolumn{2}{|c|}{$\begin{array}{l}13-130 \\
P T T^{* *}\end{array}$} \\
\hline & $1 \mathrm{~h}$ & $\mathrm{R}$ & $3 \mathrm{de}$ & MR & 3 def & MR & 4 fghi & MR-MS & $3 \mathrm{klm}$ & MR & $3 \mathrm{klm}$ & MR \\
\hline 66 & $2 \mathrm{fgh}$ & R-MR & 3 de & MR & 3 efg & MR & 6 bcde & MR-MS & $4 \mathrm{jkl}$ & MR-MS & $6 \mathrm{cdef}$ & MR-MS \\
\hline 67 & $5 \mathrm{cde}$ & MR-MS & 3 cde & MR & $5 a b c$ & MR-MS & 6 bcde & MR-MS & 6 defg & MR-MS & 4 hijk & MR-MS \\
\hline 68 & $8 \mathrm{a}$ & MS-S & $7 a$ & MS & $7 \mathrm{a}$ & MS & $7 \mathrm{a}$ & $\underline{\text { MR-MS }}$ & 6 defg & $\underline{\text { MR-MS }}$ & $7 \mathrm{abc}$ & MS \\
\hline 69 & 3 def & MR & $3 \mathrm{de}$ & MR & 3 efg & MR & 5 efgh & $\underline{\text { MR-MS }}$ & $7 \mathrm{bc}$ & $\underline{\mathrm{MS}}$ & $4 \mathrm{ijk}$ & MR-MS \\
\hline 70 & 3 efg & MR & $5 \mathrm{bcd}$ & MR-MS & 3 def & MR & 4 ghijk & MR-MS & $6 \mathrm{cdef}$ & $\underline{\mathrm{MR}}-\mathrm{MS}$ & 4 hijk & MR-MS \\
\hline 71 & $7 \mathrm{abc}$ & MS & 5 bcd & MR-MS & 3 def & MR & 4 ghij & MR-MS & $4 \mathrm{jkl}$ & MR-MS & $3 \mathrm{klm}$ & MR \\
\hline 72 & $5 \mathrm{bcd}$ & MR-MS & $5 \mathrm{bc}$ & MR-MS & $5 \mathrm{abc}$ & MR-MS & 6 bcde & $\underline{\text { MR-MS }}$ & $7 \mathrm{~cd}$ & $\underline{\mathrm{MS}}$ & $7 \mathrm{abc}$ & MS \\
\hline 73 & 3 def & MR & $5 \mathrm{bcd}$ & MR-MS & $5 \mathrm{bcd}$ & MR-MS & 6 bcde & MR-MS & 6 cde & MR-MS & 5 efgh & MR-MS \\
\hline 74 & $2 \mathrm{fgh}$ & R-MR & 3 cde & MR & 3 cde & MR & 6 bcde & $\underline{\text { MR-MS }}$ & $7 \mathrm{bc}$ & $\underline{\mathrm{MS}}$ & 5 defg & MR-MS \\
\hline 75 & $7 \mathrm{abc}$ & MS & $5 \mathrm{bc}$ & MR-MS & 3 efg & MR & 4 fghı & MR-MS & $5 \mathrm{fgh}$ & MR-MS & $4 \mathrm{ijk}$ & MR-MS \\
\hline 76 & 5 cde & MR-MS & 3 cde & MR & 3 def & MR & 6 bcde & MR-MS & 6 cde & MR-MS & $6 \mathrm{cdef}$ & MR-MS \\
\hline 77 & $7 \mathrm{abc}$ & MS & $5 \mathrm{abc}$ & MR-MS & $5 \mathrm{bcd}$ & MR-MS & 6 bcde & MR-MS & 4 hij & $\underline{\text { MR-MS }}$ & 4 hijk & MR-MS \\
\hline 78 & 3 efg & MR & 3 cde & MR & 3 cde & MR & 6 bcde & $\underline{\text { MR-MS }}$ & 6 defg & $\underline{\text { MR-MS }}$ & 5 defg & MR-MS \\
\hline 79 & $3 \mathrm{def}$ & MR & $5 \mathrm{bcd}$ & MR-MS & $5 \mathrm{bcd}$ & MR-MS & $6 \mathrm{abcd}$ & $\underline{\text { MR-MS }}$ & $7 \mathrm{bc}$ & $\underline{\mathrm{MS}}$ & 6 defg & MR-MS \\
\hline 80 & $5 \mathrm{bcd}$ & MR-MS & $5 \mathrm{bcd}$ & MR-MS & $2 \mathrm{fgh}$ & R-MR & $7 a b$ & $\underline{\mathrm{MS}}$ & $8 \mathrm{ab}$ & $\underline{\text { MS-S }}$ & 5 efgh & MR-MS \\
\hline 81 & 3 def & MR & $2 \mathrm{e}$ & R-MR & $1 \mathrm{~h}$ & $\mathrm{R}$ & 6 bcde & $\underline{\text { MR-MS }}$ & 6 defg & $\underline{\text { MR-MS }}$ & 5 defg & MR-MS \\
\hline 82 & 3 efg & MR & $5 \mathrm{bcd}$ & MR-MS & 5 bcd & MR-MS & 6 abcd & $\overline{\text { MR-MS }}$ & $7 \mathrm{~cd}$ & $\underline{\mathrm{MS}}$ & 5 defg & MR-MS \\
\hline 83 & 3 def & MR & $5 \mathrm{bcd}$ & MR-MS & 3 cde & MR & 4 fghı & $\underline{\text { MR-MS }}$ & 6 defg & $\underline{\mathrm{MR}-\mathrm{MS}}$ & $4 \mathrm{jjk}$ & MR-MS \\
\hline 84 & $2 \mathrm{fgh}$ & R-MR & $5 \mathrm{bcd}$ & MR-MS & 3 cde & MR & 4 ghij & $\overline{\text { MR-MS }}$ & $4 \mathrm{hij}$ & $\overline{\mathrm{MR}-\mathrm{MS}}$ & 2 mno & R-MR \\
\hline 85 & 5 cde & MR-MS & $3 \mathrm{de}$ & MR & 3 def & MR & 5 defg & MR-MS & $7 \mathrm{~cd}$ & MS & 6 bcde & MR-MS \\
\hline 86 & $7 \mathrm{abc}$ & MS & $7 a b$ & MS & $5 a b c$ & MR-MS & 6 bcde & MR-MS & $5 \mathrm{efg}$ & MR-MS & $7 a b$ & MS \\
\hline 87 & $5 \mathrm{bcd}$ & MR-MS & $5 \mathrm{bcd}$ & MR-MS & $5 \mathrm{bcd}$ & MR- & & $\underline{\text { MR-MS }}$ & 6 defg & MR-MS & $7 a b c$ & MS \\
\hline 88 & $5 a b c$ & MR-MS & $5 \mathrm{bc}$ & MR-MS & $5 \mathrm{abc}$ & MR-MS & $6 a b c$ & $\overline{\mathrm{MR}}-\underline{\mathrm{MS}}$ & $7 \mathrm{bc}$ & $\mathrm{MS}$ & 6 bcde & MR-MS \\
\hline 89 & 3 def & MR & $3 \mathrm{de}$ & MR & 3 def & MR & $3 \mathrm{jkl}$ & MR & $3 \mathrm{klm}$ & MR & $3 \mathrm{klm}$ & MR \\
\hline 90 & $7 \mathrm{abc}$ & MS & $5 \mathrm{abc}$ & MR-MS & $5 \mathrm{bcd}$ & MR- & 6 bcde & MR-MS & 2 no & R-M & $5 \mathrm{fgh} 1$ & MR-MS \\
\hline 91 & $7 \mathrm{abc}$ & MS & $5 \mathrm{bcd}$ & MR-MS & $5 \mathrm{bcd}$ & MR- & 4 ghij & $\underline{\text { MR-MS }}$ & $5 \mathrm{fgh}$ & MR-MS & $4 \mathrm{ijk}$ & $\underline{\mathrm{MR}}-\mathrm{MS}$ \\
\hline 92 & $7 \mathrm{ab}$ & MS & $5 a b c$ & MR-MS & $5 \mathrm{bcd}$ & MR-MS & 4 fghi & $\underline{\overline{\mathrm{MR}}}-\mathrm{MS}$ & 6 defg & MR-MS & $5 \mathrm{efgh}$ & $\overline{\mathrm{MR}}-\mathrm{MS}$ \\
\hline 93 & $7 \mathrm{abc}$ & MS & $3 \mathrm{de}$ & MR & 3 efg & MR & $3 \mathrm{ijkl}$ & MR & $4 \mathrm{jkl}$ & $\underline{\text { MR-MS }}$ & $3 \mathrm{klm}$ & MR \\
\hline 94 & 3 efg & MR & $3 \mathrm{de}$ & MR & $3 \mathrm{def}$ & MR & $3 \mathrm{jkl}$ & MR & $3 \mathrm{klm}$ & MR & 20 & R-MR \\
\hline 95 & $5 \mathrm{bcd}$ & MR-MS & $5 \mathrm{bcd}$ & MR-MS & $5 \mathrm{bcd}$ & MR-MS & $6 \mathrm{abcd}$ & MR-MS & $4 \mathrm{jkl}$ & MR-MS & 5 efgh & MR-MS \\
\hline 96 & $7 a b c$ & MS & $5 \mathrm{bc}$ & MR-MS & $5 \mathrm{bcd}$ & MR-MS & 6 bcde & MR-MS & $4 \mathrm{jjk}$ & $\underline{\mathrm{MR}}-\mathrm{MS}$ & $3 \mathrm{jkl}$ & MR \\
\hline 97 & $7 \mathrm{abc}$ & MS & $5 \mathrm{abc}$ & MR-MS & 3 cde & MR & 6 bcde & MR-MS & $6 \mathrm{defg}$ & $\overline{\mathrm{MR}-\mathrm{MS}}$ & 5 defg & MR-MS \\
\hline 98 & 5 cde & MR-MS & $5 \mathrm{bcd}$ & MR-MS & 3 def & MR & $6 \mathrm{abc}$ & MR-MS & $5 \mathrm{fgh}$ & $\underline{\text { MR-MS }}$ & 5 fghı & MR-MS \\
\hline 99 & 3 efg & MR & 3 de & MR & $1 \mathrm{~h}$ & $\mathrm{R}$ & 4 ghij & MR-MS & $1 \mathrm{p}$ & $\underline{\mathrm{R}}$ & $4 \mathrm{jjk}$ & MR-MS \\
\hline 100 & 3 def & MR & $5 \mathrm{bcd}$ & MR-MS & $3 \mathrm{def}$ & MR & 6 abcd & MR-MS & $5 \mathrm{fgh}$ & $\underline{\text { MR-MS }}$ & 4 ghij & MR-MS \\
\hline 101 & 3 def & MR & 5 cde & MR-MS & $3 \mathrm{efg}$ & MR & 6 bcde & MR-MS & $3 \mathrm{klm}$ & $\underline{\mathrm{MR}}$ & $3 \mathrm{jkl}$ & MR \\
\hline 102 & $2 \mathrm{fgh}$ & R-MR & 2 & R-MR & 2 efgh & R-MR & 5 defg & MR-MS & $3 \mathrm{lmn}$ & $\underline{\mathrm{MR}}$ & 4 ghıj & MR-MS \\
\hline 103 & 5 cde & MR-MS & $5 \mathrm{bcd}$ & MR-MS & 3 cde & MR & 6 abcd & MR-MS & $4 \mathrm{jkl}$ & $\underline{\text { MR-MS }}$ & $4 \mathrm{jjk}$ & MR-MS \\
\hline 104 & 3 efg & MR & $3 \mathrm{de}$ & MR & $2 \mathrm{fgh}$ & R-MR & 5 defg & MR-MS & $3 \mathrm{klm}$ & $\underline{\mathrm{MR}}$ & 20 & R-MR \\
\hline 105 & $5 \mathrm{cde}$ & MR-MS & $7 \mathrm{ab}$ & MS & $5 \mathrm{bcd}$ & MR-MS & $7 \mathrm{a}$ & MS & $6 \mathrm{efg}$ & MR-MS & $4 \mathrm{ijk}$ & MR-MS \\
\hline 106 & $5 \mathrm{bcd}$ & MR-MS & $5 \mathrm{bc}$ & MR-MS & $5 \mathrm{abc}$ & MR-MS & $7 \mathrm{a}$ & MS & $5 \mathrm{fgh}$ & $\underline{\text { MR-MS }}$ & $2 \mathrm{mno}$ & R-MR \\
\hline 107 & 3 def & MR & 3 cde & MR & 3 cde & MR & $7 \mathrm{ab}$ & MS & $6 \mathrm{cdef}$ & $\underline{\text { MR-MS }}$ & $4 \mathrm{jjk}$ & MR-MS \\
\hline $\mathrm{CV} \%$ & $9.41 \%$ & & $9.98 \%$ & & $9.58 \%$ & & $5.16 \%$ & & $4.46 \%$ & & $5.53 \%$ & \\
\hline
\end{tabular}

*P. teres f. maculata scale values: 1= R: resistant, 2= R: resistant to MR: moderately resistant, $3=$ MR: moderately resistant, $5=\mathrm{MR}$ : moderately resistant to MS: moderately susceptible, 7=MS: moderately susceptible, $8=$ MS: moderately susceptible to S: susceptible, $9=\mathrm{S}$ : susceptible. ${ }^{*}$ *P. teres f. teres scale values: $1=\mathrm{R}$ : resistant, $2=\mathrm{R}$ : resistant to MR: moderately resistant, $3=\mathrm{MR}$ : moderately resistant, 4= MR: moderately resistant to MS: moderately susceptible, 5= MR: moderately resistant to MS: moderately susceptible, 6= MR: moderately resistant to MS: moderately susceptible, 7= MS: moderately susceptible, $8=$ MS: moderately susceptible to S: susceptible, $9=\mathrm{S}$ : susceptible, $10=\mathrm{VS}$ : very susceptible 
$H$. spontaneum and $300 \mathrm{H}$. vulgare accessions were evaluated for their resistance status to $P$. teres under greenhouse and field conditions. H. spontaneum genotypes exhibited different resistance reactions and 143 genotypes showed resistant reaction to all isolates. Fetch et al (2003) determined the diversity of $116 \mathrm{H}$. spontaneum genotypes for their reaction to six barley fungal pathogens. The genotypes were obtained from Israel and Jordan. At seedling stage, a high level of diversity was found. Resistance frequency of genotypes from Israel and Jordan was high for net blotch (68\% and $72 \%$, respectively). Two genotypes were found resistant to 6 pathogens. Similarly, in our current study variation was found among the $H$. spontaneum genotypes. In our study, six $H$. spontaneum genotypes showed resistant to moderately resistant reactions to all $\mathrm{Ptt}$ and $\mathrm{Ptm}$ isolates. Jana \& Bailey (1995) determined the resistance status of $H$. vulgare subsp. spontaneum and $H$. vulgare subsp. vulgare genotypes from Jordan and Turkey to P. teres f. maculata, P. teres f. teres and Cochliobolus sativus. More $H$. vulgare subsp. spontaneum genotypes were resistant to P. teres f. teres $(21.8 \%$ vs. $0.5 \%)$ than $H$. vulgare subsp. vulgare. An equal number of $H$. vulgare subsp. spontaneum and $H$. vulgare subsp. vulgare genotypes were resistant to $P$. teres f. maculata. A larger percentage of $H$. vulgare subsp. spontaneum genotypes $(10.5 \%)$ had at least moderate resistance to $P$. teres f. teres, $P$. teres f. maculata and $C$. sativus compared to only $1.3 \%$ in $H$. vulgare subsp. vulgare. However, in our current study, $25 \%$ of the genotypes and $7.6 \%$ of the genotypes exhibited resistant group reactions to $P$. teres f. maculata and $P$. teres f. teres, respectively. This finding is hopeful, because $P$. teres f. maculata is more prevalent in Turkey than P. teres f. teres (Karakaya et al 2014). H. spontaneum accessions showed different resistance reactions, depending upon their origin. Sato \& Takeda (1997) evaluated net blotch resistance in $175 \mathrm{H}$. vulgare subsp. spontaneum (H. spontaneum) accessions and 149 wild Hordeum accessions of thirteen species or subspecies. Most $H$. spontaneum accessions showed resistance to each of the four P. teres f. teres isolates (Japanese isolates $\mathrm{K} 105$ and Pt860514 and Canadian isolates
WRS102 and WRS1581) tested. Some accessions from Russia and Afghanistan showed a high level of resistance and Morocco accessions were susceptible. H. spontaneum accessions susceptible to the Canadian isolate WRS102 but resistant to the other three isolates were found in Iraq. This suggested the geographical differentiation of resistance genes in H. spontaneum. All accessions of the other wild Hordeum species, especially some accessions of $H$. marinum subsp. gussoneanum, showed high levels of resistance. Sato \& Takeda (1997) concluded that resistance genes may be useful candidates for incorporation into cultivated barley.

$H$. spontaneum is a rich source of genes for disease resistance. Many resistant barley genotypes were found in barley evolution centers (Afanasenko et al 2000). Suitable habitat conditions for $H$. vulgare subsp. spontaneum exist especially in the Levant and Turkey and genetic diversity was observed in these populations (Jakob et al 2014). Turkey is an important gene center of barley and wild barleys (Kün 1996). Karakaya et al (2016) examined a total of 40 naturally growing $H$. spontaneum field populations in Şanlıurfa, Mardin, Şırnak, Siirt, Diyarbakır, Gaziantep, Kilis and Hatay provinces of Turkey for the presence of diseases and their severities in 2015 . Nine $H$. spontaneum populations were disease free. The following diseases were found: Scald incited by Rhynchosporium commune, powdery mildew incited by Blumeria graminis f. sp. hordei, both forms of net blotch incited by Drechslera teres f. teres and D. teres f. maculata, semi loose smut incited by Ustilago nigra, loose smut incited by Ustilago nuda, brown rust (leaf rust) incited by Puccinia hordei and barley stripe caused by Drechslera graminea. Scald was the most commonly encountered disease followed by powdery mildew and net blotch. The incidence and severity values of diseases varied. The authors reported a wide range of variation in terms of disease resistance status of naturally growing H. spontaneum populations. 


\section{Conclusions}

The use of disease resistant cultivars is the desirable control method of diseases. For sustainable crop production, monitoring virulence changes in pathogen is necessary. New pathotypes could be more virulent than previous pathotypes. For this reason, a broad base of genetical source is necessary. Wild barleys and especially $H$. spontaneum are valuable sources for disease resistance. Useful traits including disease resistance could be transferred to barley cultivars. Nevo (1992) pointed out the importance of $H$. spontaneum for disease resistant barley breeding programs and for developing a gene pool for desired traits. Also, Nevo et al (1986) examined the $H$. spontaneum populations of Israel, Turkey and Iran in the Fertile Crescent and reported their genetic diversity as well as their adaptability. Turkey is an important gene center of Hordeum species (Kün 1996). Hordeum spontaneum populations are naturally growing in Turkey and heterogenous nature of disease resistance among the populations has been reported (Karakaya et al 2016).

With this study, novel wild barley (H. spontaneum) genotypes resistant to both forms of Pyrenophora teres have been identified. These genotypes could be used in obtaining disease resistant and high yielding barley cultivars.

\section{Acknowledgements}

This study was financially supported by The Scientific and Technological Research Council of Turkey (Project No: 1110644). We thank Sinan AYDOĞAN for his help on statistical analysis.

\section{References}

Afanasenko O S, Makarova I G \& Zubkovich A A (2000). Inheritance of resistance to different Pyrenophora teres Dreschs. strains in barley accession CI 5791. In: Logue S. (Ed.). Proceedings of $8^{\text {th }}$ International Barley Genetics Symposium, 22-27 October, Adelaide, Australia, 2: 73-75
Aktaş H (1995). Reaction of Turkish and German barley varieties and lines to the virulent strain T4 of Pyrenophora teres. Rachis 14: 9-13

Ceccarelli S \& Grando S (2000). Barley landraces from the Fertile Crescent. A lesson for plant breeders. In: S B Brush (Ed.), Genes in the field, On-farm conservation of crop diversity. Int. Plant Gen. Res. Institute, International Developmet Research Center, Lewis Publishers, Boca Raton London New York Washington, D.C. pp. 51-76

Çelik E \& Karakaya A (2017). Yabani arpa (Hordeum spontaneum) ve hastal1klara dayan1kl1l1k. Mustafa Kemal Üniversitesi Ziraat Fakültesi Dergisi 22: 6586

Çelik Oğuz A (2015). Determination of the pathotypes of Pyrenophora teres in Turkey and assessment of the reactions of some barley landraces and wild barley (Hordeum spontaneum) populations to net blotch. $\mathrm{PhD}$ Thesis, Graduate School of Natural and Applied Sciences, Ankara University, Turkey (In Turkish)

Çelik Oğuz A, Karakaya A, Duran R M \& Özbek K. (2017). Net blotch resistant Hordeum spontaneum genotypes identified. 2017. International Workshop Plant Health: Challenges and Solutions Poster Abstracts 23-28 April, Antalya, Turkey

Douiyssi A, Rasmusson D C \& Roelfs A P (1998). Responses of barley cultivars and lines to isolates of Pyrenophora teres. Plant Disease 82: 316-321

Fetch, Jr, T G, Steffenson B J \& Nevo E (2003). Diversity and sources of multiple disease resistance in Hordeum spontaneum. Plant Disease 87: 1439-1448

Jakob S S, Rödder D, Engler J O, Shaaf S, Özkan H, Blattner F R \& Kilian B (2014). Evolutionary history of wild barley (Hordeum vulgare subsp. spontaneum) analyzed using multilocus sequence data and paleodistribution modeling. Genome Biology and Evolution 6: 685-702

Jana S \& Bailey K L (1995). Responses of wild and cultivated barley from West Asia to net blotch and spot blotch. Crop Science 35: 242-246

Karakaya A \& Akyol A (2006). Determination of the seedling reactions of some Turkish barley cultivars to the net blotch. Plant Pathology Journal 5: 113-114

Karakaya A, Mert Z, Çelik Oğuz A, Azamparsa M R, Çelik E, Akan K \& Çetin L (2014). Current status of scald and net blotch diseases of barley in Turkey. IWBLD-1st International Workshop on Barley Leaf Diseases, June 3-6, Salsomaggiore Terme, Italy 
Karakaya A, Mert Z, Çelik Oğuz A, Ertaş M N \& Karagöz A (2016). Determination of the diseases occurring on naturally growing wild barley (Hordeum spontaneum) field populations. Works of the Faculty of Agriculture and Food Science, University of Sarajevo 61, 66/1: 291-295

Kopahnke D (1998). Evalution of barley for resistance to Drechslera teres (Sacc.) Shoem. Beiträge zur Züchtungsforschung-Bundesansalt für Züchtungsforschung an Kulturpflanzen 4: 1-3

Kün E (1996). Tahıllar-1 (Serin İklim Tahılları). Ankara Üniversitesi Ziraat Fakültesi Yayınları, Yayın No: 1451, Ankara

Liu Z, Ellwood S R, Oliver R P \& Friesen T L (2011). Pyrenophora teres: profile of an increasingly damaging barley pathogen, Molecular Plant Pathology 12: 1-19

Mathre D E (Ed.) (1982). Compendium of Barley Diseases. APS Press. Minnesota,78 pp

Nevo E (1992). Origin, evolution, population genetics and resources for breeding of wild barley, Hordeum spontaneum, in the Fertile Crescent. In: Shewry, P. R. (Ed.). Barley: genetics, biochemistry, molecular biology and biotechnology. C.A.B. International. UK, pp. 19-43

Nevo E (2012). Evolution of wild barley and barley improvement. In: Advance in Barley Sciences. C. Li, G. Zhang, X. Liu and J. Eglinton (Eds.). Proceedings of $11^{\text {th }}$ International Barley Genetics Symposium. Zhejiang University Press- Springer, pp. 1-16
Nevo E, Beiles A \& Zohary D (1986). Genetic resources of wild barley in the Near East: structure, evolution and application in breeding. Biological Journal of the Linnean Society 24: 355-380

Sato K \& Takeda K (1997). Net blotch resistance in wild species of Hordeum. Euphytica 95: 179-185

Taşkoparan H \& Karakaya A (2009). Assessment of the seedling reactions of some barley cultivars to Drechslera teres f. maculata. Selçuk Tarım ve Gida Bilimleri Dergisi 23(50): 60-62

Tekauz A (1985). A numerical scale to classify reactions of barley to Pyrenophora teres. Canadian Journal Plant Pathology 7: 181-183

Usta P, Karakaya A, Çelik Oğuz A, Mert Z, Akan K \& Çetin L (2014). Determination of the seedling reactions of twenty barley cultivars to six isolates of Drechslera teres f. maculata. Anadolu Tartm Bilimleri Dergisi 29: 20-25

Vavilov N I (1951). The origin, variation, immunity and breeding of cultivated plants, (translated from the Russian by K. S. Chester). Chronica Botanica; New York: Stechert-Hafner

Yazıcı B, Karakaya A, Çelik Oğuz A \& Mert Z (2015). Determination of the seedling reactions of some barley cultivars to Drechslera teres f. teres. Bitki Koruma Bülteni 55: 239-245

Zadoks J C, Chang T T \& Konzak C F (1974). A decimal code for the growth stages of cereals. Weed Research 14: 415- 421 